\begin{tabular}{|c|l|}
\hline Title & Theoretical study on resonant transmission of acoustic phonons propagating through a superlattice liquid interface \\
\hline Author(s) & Mizuno, Seiji \\
\hline Citation & $\begin{array}{l}\text { Physical Review B, 63(3), 035301 } \\
\text { https://doi.org/10.1103/PhysRevB.63.035301 }\end{array}$ \\
\hline Issue Date & 2000-12-22 \\
\hline Doc URL & http://hdl.handle.net/2115/5706 \\
\hline Rights & Copyright $\odot 2000$ A merican Physical Society \\
\hline Type & article \\
\hline File Information & PRB63.pdf \\
\hline
\end{tabular}

Instructions for use 


\title{
Theoretical study on resonant transmission of acoustic phonons propagating through a superlattice-liquid interface
}

\author{
Seiji Mizuno \\ Department of Applied Physics, Hokkaido University, Sapporo 060-8628, Japan
}

(Received 17 July 2000; published 22 December 2000)

\begin{abstract}
We theoretically studied the resonant interaction of acoustic phonons with the vibrational mode localized near a superlattice-liquid interface. We calculated the phonon transmission rate and examined the peculiar resonance peak due to the localized mode. The peak value depends on the number of periods $(N)$ of the superlattice, and this resonant feature disappears for large $N$ and also small $N$. We derived the formula describing the resonance line and discussed the condition for such a resonance to be seen.
\end{abstract}

DOI: 10.1103/PhysRevB.63.035301

PACS number(s): 68.65.-k, 62.65.+k, 63.20.-e

\section{INTRODUCTION}

The theoretical investigation of phonon properties in the superlattice (SL) starts with the calculation of the phonondispersion relations. ${ }^{1,2}$ The outline of the dispersion relation of the SL can be understood by folding the dispersion curves for a bulk solid into the mini-Brillouin zone (BZ) of the SL. ${ }^{1}$ In this folded dispersion relation, moreover, the frequency gaps are generated at the center and edges of the mini-BZ due to the Bragg reflection. The size of the mini-BZ is determined by the length of the unit period of the SL. Thus, we can set the frequency gaps in an experimentally accessible frequency range $(\sim$ sub-THz), changing the unit period of the SL. Actually, the existence of the frequency gaps was experimentally confirmed for the GaAs/AlAs SL, etc. with the use of phonon spectroscopy. ${ }^{2-4}$

In the calculation of the dispersion relations, the perfect periodicity should be assumed and the periodic boundary condition is applied. On the other hand, in the realistic SL, various kinds of inhomogeneity are embedded. The most typical example is a free surface or a defect layer, which causes localized vibrational modes inside the frequency gaps. Theoretically, the impurity and surface vibrational modes have been studied for the infinite and semi-infinite SL's, respectively. ${ }^{5,6}$ Experimentally, the surface modes have been detected in various SL's by using the picosecond ultrasonic technique ${ }^{7}$ and Raman scattering experiments. ${ }^{8}$ The impurity-layer mode is also suggested in Raman spectrum. ${ }^{9}$

Very recently, the effect of another inhomogeneity, such as the size effect or internal surface (substrate-SL or detector-SL interface), has been discussed theoretically. ${ }^{10-12}$ Actually, the SL is grown on the substrate (and often covered with a cap layer or detector layer) and the number of periods $(N)$ of the SL is finite $\left(\sim 10^{2}\right)$, which is much smaller than that of a bulk solids $\left(\sim 10^{8}\right)$. Thus, in some case the effects of these become important for the realistic SL. It is relevant to calculate the phonon transmission or reflection rate for such a finite-size SL grown on a substrate.

In 1999 Kato $^{12}$ calculated the phonon transmission rate of the GaAs/AlAs SL in contact with a distilled water, numerically. His calculation shows that the resonance peak exists in the frequency gap. This resonance was attributed to the sur- face vibrational mode. However, strictly speaking, the system he considered has an interface (between the SL and the liquid) and not a free surface. In addition, we should notice the serious problem related to the conservation of energy. The phonon displacement corresponding to the surface mode decays exponentially away from the surface. Conversely, the displacement of the phonon injected from the substrate increases in SL exponentially, i.e., the energy flux of the phonon is amplified in the SL. This means that we can extract a higher energy than that of the incident phonon. Clearly, more detailed investigation is necessary.

In the present paper we study the transmission rate of the phonons from the SL into liquid, based on the rigorous formula. In particular, we examine the $N$ dependence of the resonant feature and give the physical explanation. In Sec. II we briefly summarize the general expression for the transmission rate of phonons propagating through the periodic $\mathrm{SL}$, which is used for the later analysis. As a numerical example, in Sec. III we apply this analytical formula to the GaAs- $(\mathrm{GaAs} / \mathrm{AlAs})_{N^{-}} \mathrm{H}_{2} \mathrm{O}$ and $\mathrm{GaAs}-(\mathrm{GaAs} / \mathrm{AlAs})_{N^{-}}{ }^{4} \mathrm{He}$ systems. The result Kato calculated numerically is also reproduced in this section. In Sec. IV we explain the main feature of the frequency dependence of the transmission rate in these systems. In particular, the necessary conditions for the resonance to be seen are examined. In Sec. V we discuss the peculiar resonant properties of phonons propagating through the SL-liquid (SL-L) interface in detail. A summary and conclusions are given in Sec. VI.

\section{GENERAL EXPRESSION OF THE PHONON TRANSMISSION RATE}

A schematic picture of the situation we study is illustrated in Fig. 1. In order to define the transmission rate, the existence of the substrate $(S)$ and detector $(D)$ is indispensable; this fact becomes important in the present study. Here, we consider the case where the wave vector of phonons is perpendicular to the interfaces of the layer. In this case, three phonon modes are decoupled from each other if the interfaces are a mirror-symmetry plane. That is, we can treat only one mode, e.g., the longitudinal mode. Also, the continuum model which should be valid for sub-THz phonons is assumed for each constituent layer. The displacement field of incident phonons in the substrate is simply represented by 


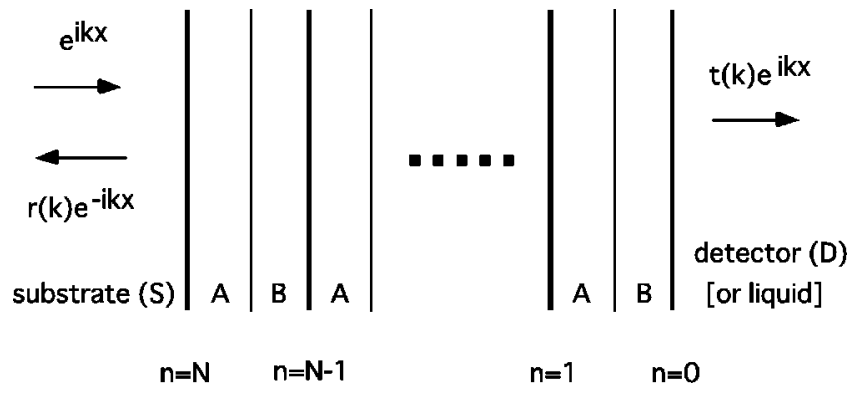

FIG. 1. Schematic of the superlattice system.

the plane wave $e^{i k x}$ of unit amplitude. The incident phonon is multiple scattered from each interface. After the scattering, the displacement fields of the transmitted and reflected phonons in the substrate and the detector layer are expressed as $t(k) e^{i k x}$ and $r(k) e^{-i k x}$, respectively. Here, $t(k)$ and $r(k)$ are the transmission and reflection amplitudes, respectively.

The energy flux of incident and transmitted phonons are given by

$$
\begin{gathered}
P_{S}=\frac{1}{2} \rho_{S} \omega^{2} v_{S}=\frac{1}{2} \omega^{2} Z_{S}, \\
P_{D}=\frac{1}{2} \rho_{D} \omega^{2} v_{D}|t(k)|^{2}=\frac{1}{2} \omega^{2} Z_{D}|t(k)|^{2},
\end{gathered}
$$

respectively. ${ }^{2}$ Here, $\omega$ is the angular frequency of the phonon and $Z_{i}=\rho_{i} v_{i}(i=S, D)$ is the acoustic impedance given by the product of the mass density $\rho_{i}$ and the sound velocity $v_{i}$. The transmission rate is defined as the ratio of these energy fluxes,

$$
T=\frac{P_{D}}{P_{S}}=\frac{Z_{D}}{Z_{S}}|t(k)|^{2} .
$$

For the periodic SL, the analytical expression of the phonon transmission rate is given in our previous paper. ${ }^{13}$ The result is

$$
T=\frac{4 \frac{Z_{D}}{Z_{S}}}{\left(\frac{Z_{D}}{Z_{A}} T_{12}-\frac{Z_{A}}{Z_{S}} T_{21}\right)^{2}+\left(T_{22}+\frac{Z_{D}}{Z_{S}} T_{11}\right)^{2}},
$$

where $T_{i j}$ is the matrix elements of transfer matrix for the periodic SL,

$$
\begin{gathered}
T_{11}=\frac{\lambda-\mu}{2} S(N)+C(N), \\
T_{12}=\sigma S(N), \\
T_{21}=\zeta S(N), \\
T_{22}=-\frac{\lambda-\mu}{2} S(N)+C(N),
\end{gathered}
$$

where

$$
S(N)=\left(\frac{\mu+\lambda}{|\mu+\lambda|}\right)^{N+1} \frac{\sinh N \theta}{\sinh \theta},
$$

$$
\begin{gathered}
C(N)=\left(\frac{\mu+\lambda}{|\mu+\lambda|}\right)^{N} \cosh N \theta, \\
\cosh \theta \equiv\left|\frac{\mu+\lambda}{2}\right|,
\end{gathered}
$$

and

$$
\begin{aligned}
& \lambda=\cos k_{A} d_{A} \cos k_{B} d_{B}-\frac{Z_{A}}{Z_{B}} \sin k_{A} d_{A} \sin k_{B} d_{B}, \\
& \sigma=\sin k_{A} d_{A} \cos k_{B} d_{B}+\frac{Z_{A}}{Z_{B}} \cos k_{A} d_{A} \sin k_{B} d_{B} \\
& \zeta=-\sin k_{A} d_{A} \cos k_{B} d_{B}-\frac{Z_{B}}{Z_{A}} \cos k_{A} d_{A} \sin k_{B} d_{B}, \\
& \mu=\cos k_{A} d_{A} \cos k_{B} d_{B}-\frac{Z_{B}}{Z_{A}} \sin k_{A} d_{A} \sin k_{B} d_{B} .
\end{aligned}
$$

In these equations $k_{i}=\omega / v_{i}$ is the wave number and $d_{A}$ and $d_{B}$ are the thicknesses of $A$ and $B$ layers, respectively. The above expressions (9)-(11) are applicable to phonons inside the frequency gap of the superlattice. Equation (4) shows clearly that the transmission rate depends on the acoustic properties of the substrate and detector through $Z_{S}$ and $Z_{D}$.

\section{NUMERICAL EXAMPLE FOR GaAs-(GaAs/AlAs)SL-H ${ }_{2} \mathrm{O}$ SYSTEM}

As the next step, we apply the above formula to the GaAs/ AlAs SL grown on the GaAs substrate and being in contact with $\mathrm{H}_{2} \mathrm{O}$. That is, $S=\mathrm{GaAs}$ and $D=\mathrm{H}_{2} \mathrm{O}$ in Fig. 1 . The frequency dependence of the transmission rate is shown in Fig. 2. Here, the number of periods $N$ is assumed to be 8 (bold line) and 16 (broken line). There are two possibilities for the stacking order of constituent layers, i.e., (a) $A$ $=$ AlAs, $B=\mathrm{GaAs}$, or (b) $A=\mathrm{GaAs}, B=\mathrm{AlAs}$. The calculated result is quite different with the stacking order. The solid line $(N=8)$ in Fig. 2(b) reproduces the previous result. $^{12}$

In both cases, the transmission rate oscillates around $T_{S \leftrightarrow D}=4 Z_{S} Z_{D} /\left(Z_{S}+Z_{D}\right)^{2}$, which is the transmission rate for the phonon propagating directly from the substrate to the detector (i.e., the single interface case). The most noticeable feature is the structure within the frequency gaps. In general, the transmission rate in the frequency gap becomes considerably small due to the Bragg reflection, i.e., the phonon displacement decays exponentially away from the interface between the substrate and SL. However, there exist sharp peaks in the first and third frequency gaps in Fig. 2(b). On the other hand, there is no such a peak in the second gap in Fig. 2(b) and all gaps in Fig. 2(a). The origin of these peaks will be examined in Sec. IV by analyzing the exact formula of the transmission rate given in Sec. II.

Another noticeable feature is that the peak value depends on $N$. The $N$ dependence was not studied by Kato. ${ }^{12}$ Figure 3(a) shows the $N$ dependence of the peak value in the lowest gap. It has the maximum value at $N=8$. For comparison, the 

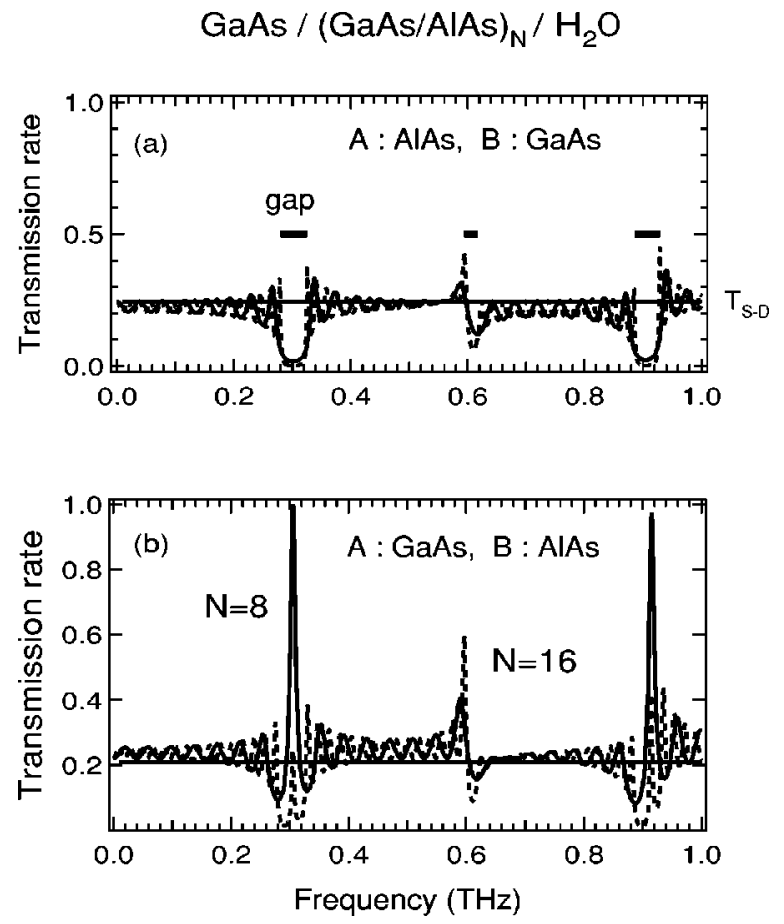

FIG. 2. Frequency dependence of the transmission rate of the longitudinal phonons in the GaAs- $(\mathrm{GaAs} / \mathrm{AlAs})_{N}-\mathrm{H}_{2} \mathrm{O}$ system with $N=8$ (solid line) and $N=16$ (dashed line): (a) $A=\mathrm{AlAs}$ and $B$ $=\mathrm{GaAs} ;$ (b) $A=\mathrm{GaAs}$ and $B=\mathrm{AlAs}$. The unit period is $(\mathrm{GaAs})_{15}(\mathrm{AlAs})_{15}$.

result calculated for the GaAs- $(\mathrm{GaAs} / \mathrm{AlAs})_{N^{-}}{ }^{4} \mathrm{He}$ system is also shown in Fig. 3(b). In this case, it has the maximum value at $N=20$. The physical interpretation is given in Sec. V.

\section{MATHEMATICAL ANALYSIS OF THE RESONANT TRANSMISSION}

In this section we explain the resonant properties shown in Sec. III, analyzing the exact expression for the transmission rate. First, we examine the necessary conditions for the resonance peak to be seen in the frequency gap and derive the approximated expression for such a resonance line.

A. Necessary conditions for the striking peak to be seen

Equation (4) can be rewritten as

$$
T=\frac{4 \gamma}{\gamma^{2}\left(T_{11}^{2}+T_{12}^{2}\right)+T_{21}^{2}+T_{22}^{2}+2 \gamma}
$$

where $\gamma=Z_{D} / Z_{A}$. Here we used the relation, $T_{11} T_{22}$ $-T_{12} T_{21}=1$, which is the general feature of the transfer matrix. To avoid unnecessary complications, we assumed in Eq. (16) that the substrate is composed of the same material as layer $A$, i.e., $Z_{S}=Z_{A}$. A necessary condition for the striking peak to be seen is given by $T \sim 1$ inside the frequency gaps. Since the terms in the denominator of Eq. (16) are all positive, this condition leads to the equations
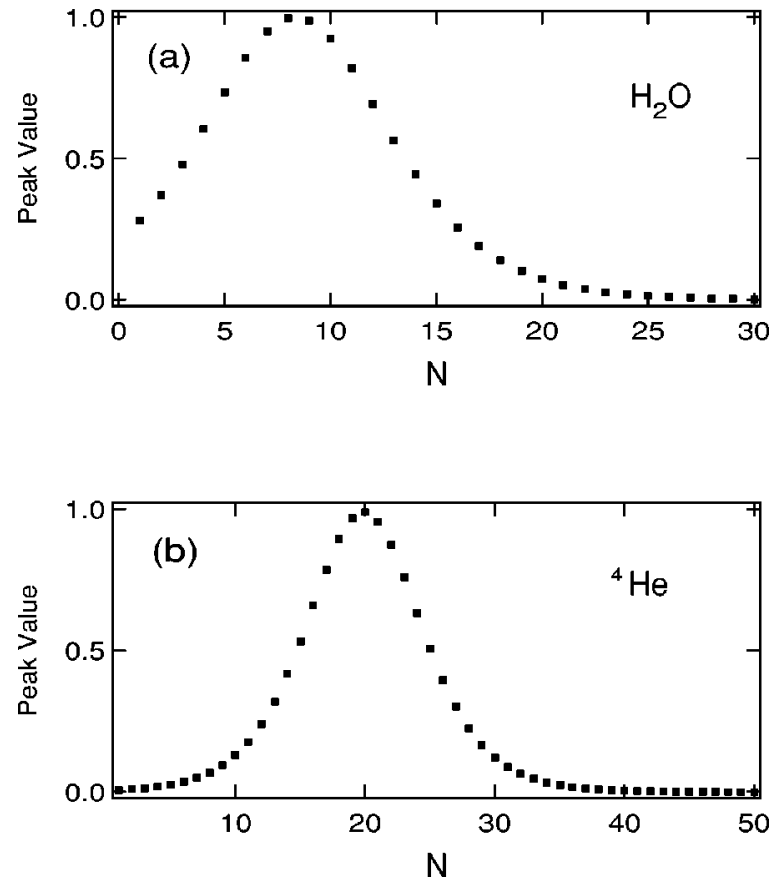

FIG. 3. $N$ dependence of the peak value in the lowest gap in (a) $\mathrm{GaAs}-(\mathrm{GaAs} / \mathrm{AlAs})_{N^{-}} \mathrm{H}_{2} \mathrm{O}$ and (b) GaAs- $(\mathrm{GaAs} / \mathrm{AlAs})_{N^{-}}{ }^{4} \mathrm{He}$ systems.

$$
\begin{gathered}
\gamma^{2} T_{11}^{2}=\gamma^{2}\left(\frac{\lambda-\mu}{2} S(N)+C(N)\right)^{2} \lesssim \gamma \\
\gamma^{2} T_{12}^{2}=\gamma^{2} \sigma^{2} S(N)^{2} \lesssim \gamma \\
T_{21}^{2}=\zeta^{2} S(N)^{2} \lesssim \gamma \\
T_{22}^{2}=\left(-\frac{\lambda-\mu}{2} S(N)+C(N)\right)^{2} \lesssim \gamma
\end{gathered}
$$

should be satisfied simultaneously.

Now we are considering the case of $\gamma \ll 1$ because the detector layer $D$ is assumed to be liquid, whose acoustic impedance is in general much smaller than that of a solid. Thus, Eq. (19) leads to $|\zeta| \ll 1$ or $\zeta \sim 0$, because $|S(N)|$ $=\sinh N \theta / \sinh \theta>N$. In the previous work, ${ }^{14}$ it is shown that the equation $\zeta=0$ gives the eigenfrequency $\omega_{s}$ of the surface localized mode. That is, there is the possibility that $T \sim 1$ is realized only for the frequency near $\omega_{s}$. This eigenfrequency is expressed as ${ }^{14}$

$$
\omega_{s}=\omega_{m}+\frac{\varepsilon}{2 \pi} \omega_{1} \sin \left(2 \omega_{m} d_{A} / v_{A}\right)
$$

where $\omega_{m}=m \omega_{1}=m \pi\left(d_{A} / v_{A}+d_{B} / v_{B}\right)^{-1}$ is the $m$ th order Bragg frequency and $\varepsilon=Z_{B} / Z_{A}-1$ is the acoustic mismatch between the constituent layers $A$ and $B$. For most of the SL's $Z_{A}$ is close to $Z_{B}$, i.e., $|\varepsilon| \ll 1$. In the derivation of Eq. (21), more than the second-order terms of $\varepsilon$ are neglected.

From Eq. (20), we can obtain the equation 


$$
\frac{\varepsilon}{|\varepsilon|}\left|\sin \left(\omega d_{A} / v_{A}\right)\right| \sim-1
$$

Therefore, $\varepsilon$ should be negative, i.e.,

$$
Z_{B}<Z_{A}
$$

and also

$$
\left|\sin \left(\omega_{m} d_{A} / v_{A}\right)\right| \sim 1
$$

should be satisfied. For the SL with the free surface, Eq. (23) is a necessary condition for the existence of surface-localized modes. ${ }^{5,14}$ Also, Eq. (24) means that the phonon amplitude is well localized near the surface. ${ }^{14}$

The remaining conditions, Eqs. (17) and (18), give the limitation of $N$. This is shown by deriving the approximated expression of Eq. (16) with the use of the conditions (23) and (24).

\section{B. Approximated expression for the resonance line}

Using Eq. (24) we can derive the approximated expressions of the elements of the transfer matrix in the $m$ th frequency gap,

$$
\begin{gathered}
T_{11} \cong(-1)^{m N} e^{-N \varepsilon}, \\
T_{22} \cong(-1)^{m N} e^{N \varepsilon}, \\
T_{12} \cong(-1)^{m N} \frac{\pi}{\omega_{1}}\left(\omega-\omega_{m}\right) \frac{\sinh N \varepsilon}{\varepsilon}, \\
T_{21}=T_{12} .
\end{gathered}
$$

Inserting Eqs. (25)-(28) into Eq. (16), we have the resonant form

$$
T=\frac{4 \gamma}{\left(\gamma e^{-N \varepsilon}+e^{N \varepsilon}\right)^{2}+(1+\gamma)^{2} \frac{\pi^{2}}{\omega_{1}^{2}}\left(\omega-\omega_{m}\right)^{2} \frac{\sinh ^{2} N \varepsilon}{\varepsilon^{2}}} .
$$

Within the present approximation, the resonant frequency is the same as the eigenfrequency of the surface localized mode. This is also equal to the Bragg frequency $\omega_{m}$ because the second term of the right-hand side of Eq. (21) is negligible under the condition Eq. (24). From Eq. (29), it is easy to find that $T\left(\omega_{m}\right) \sim 1$ if the relation

$$
\gamma=\frac{Z_{D}}{Z_{S}} \sim e^{2 N \varepsilon}
$$

or

$$
N \sim \frac{1}{2 \varepsilon} \ln \frac{Z_{D}}{Z_{S}}=\frac{1}{2|\varepsilon|} \ln \frac{Z_{S}}{Z_{D}}
$$

is satisfied. Then, we can get the well-known Breit-Wigner form
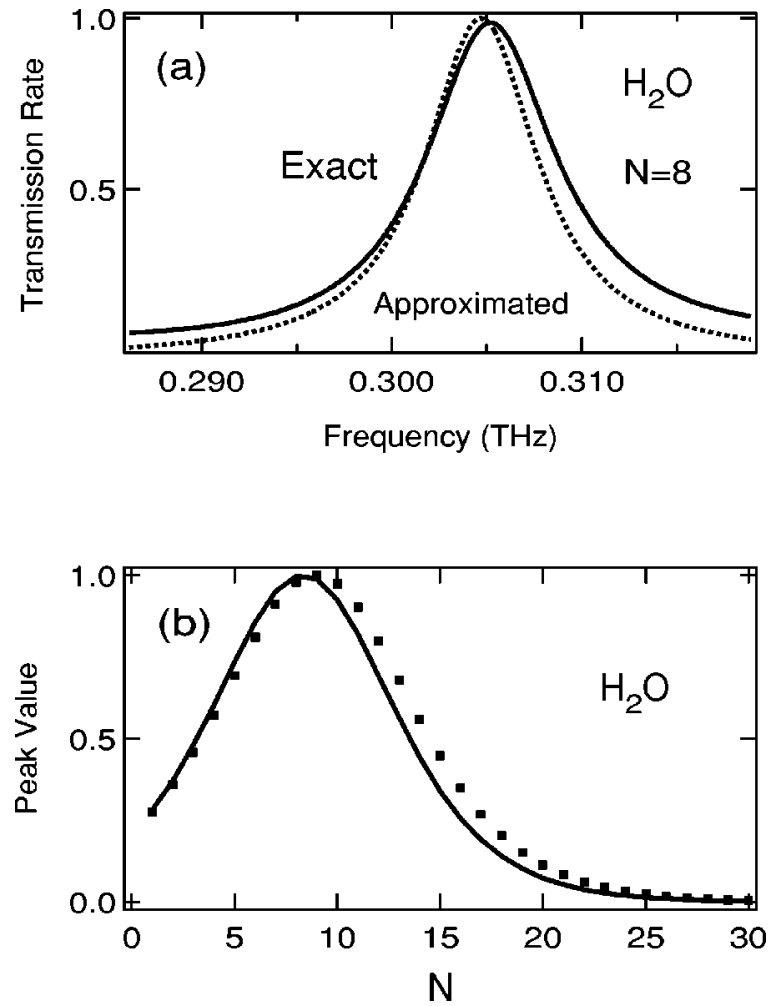

FIG. 4. (a) Phonon transmission rate versus frequency around the lowest-frequency gap in the GaAs-(GaAs/AlAs) ${ }_{N}-\mathrm{H}_{2} \mathrm{O}$ system. (b) $N$ dependence of the peak value in the lowest gap. The solid line and dots are transmission rate calculated based on the exact and approximated formulas, respectively.

$$
T=\frac{\Gamma^{2}}{\Gamma^{2}+\left(\omega-\omega_{m}\right)^{2}},
$$

where the peak width is expressed as

$$
\Gamma=\frac{4 \varepsilon \omega_{1}}{\pi}\left(\gamma^{-1}-\gamma\right)^{-1}=\frac{4 \varepsilon \omega_{1}}{\pi}\left(\frac{Z_{A}}{Z_{D}}-\frac{Z_{D}}{Z_{A}}\right)^{-1} .
$$

For the system studied in Sec. II, the frequency dependence of the transmission rate calculated from the formulas (32) and (33) is compared with the exact result in Fig. 4(a). In addition, the $N$ dependence of the peak value calculated from Eq. (29) is compared with the exact result in Fig. 4(b). Our approximated results are in good agreement with the exact one. As another example, the results calculated for the GaAs-(GaAs/AlAs) ${ }_{N^{-}}{ }^{4} \mathrm{He}$ system are also shown in Figs. 5(a) and 5(b). For the liquid whose acoustic impedance is smaller, the maximum peak value is realized for the larger $N$, according to Eq. (31).

Equations (32) and (33) are calculated for the case where $Z_{S}=Z_{A}$, but these can be generalized for $Z_{S} \neq Z_{A}$. In that case, Eq. (33) is replaced by

$$
\Gamma=\frac{4 \varepsilon \omega_{1}}{\pi}\left(\frac{Z_{S}}{Z_{A}}-\frac{Z_{A}}{Z_{S}}+\frac{Z_{A}}{Z_{D}}-\frac{Z_{D}}{Z_{A}}\right)^{-1}
$$



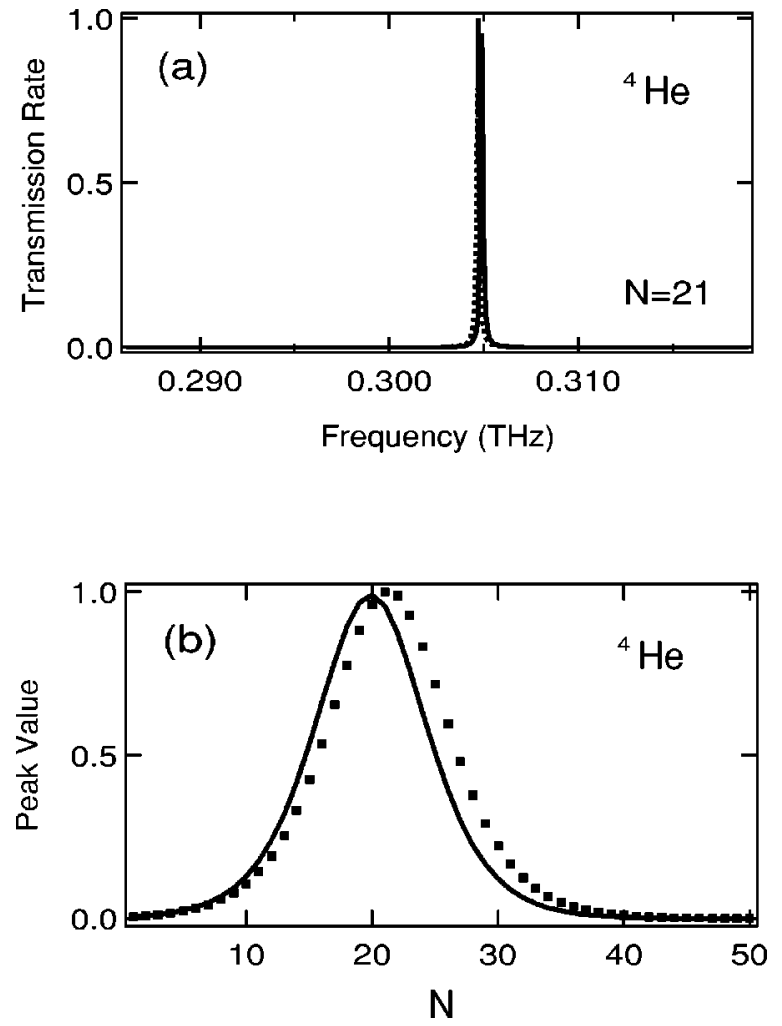

FIG. 5. (a) Phonon transmission rate versus frequency around the lowest-frequency gap in the GaAs- $(\mathrm{GaAs} / \mathrm{AlAs})_{N^{-}}{ }^{4} \mathrm{He}$ system. (b) $N$ dependence of the peak value in the lowest gap. The solid line and dots are the transmission rate calculated based on the exact and approximated formulas, respectively.

Furthermore, we can easily show that Eqs. (32) and (34) are applicable to the case where the phonons are injected from the liquid.

\section{DISCUSSIONS}

In Sec. IV we have shown that the resonance peak appears in the frequency gap, when the conditions Eqs. (23), (24), and (31) are simultaneously satisfied. To study the physical meaning of these conditions, we first consider the SL being in contact with the vacuum region (i.e., the SL has a free surface instead of the SL-L interface). As mentioned before, Eq. (23) is a necessary condition for the existence of surface-localized modes in the SL with a free surface., ${ }^{5,14}$ These modes correspond to the Wallis modes of the semiinfinite one-dimensional chain with two atoms per unit cell. ${ }^{15-17}$ It is well known that the Wallis mode appears when the atom at the end has smaller mass than the other atom. The heavier atom next to the lighter atom being at the end cannot follow the faster vibration of the lighter atom. As a result, the corresponding phonon amplitude decays exponentially away from the end. This explanation is similarly applicable to the present system.

Equation (24) means that the phonon amplitude is well localized near the surface because the corresponding phonon amplitude is expressed as ${ }^{14}$

$$
\begin{gathered}
U_{n}=\left[\mu\left(\omega_{m}\right)\right]^{n} U_{0}, \\
\mu\left(\omega_{m}\right)=(-1)^{m}\left[1+\varepsilon \sin ^{2}\left(\omega_{m} d_{A} / v_{A}\right)\right] .
\end{gathered}
$$

Here, $U_{n}$ is the phonon amplitude at the $n$th interface ( $n$ $=0$ denotes the surface). If Eq. (24) is not satisfied, i.e., $\left|\sin \left(\omega_{m} d_{A} / v_{A}\right)\right| \sim 0$, phonon amplitude hardly decays. That is, the corresponding localized vibration behaves as a bulk mode in the frequency band. In other words, the existence of the well-localized surface modes is a necessary condition for the resonance enhancement in transmission.

In the SL being in contact with liquid, this situation remains intact if the third condition (31) is satisfied. The result shown in Fig. 2(a) is calculated for the system in which Eq. (23) is not satisfied. In fact, no sharp peak can be seen in the frequency gaps. On the other hand, condition (23) is satisfied in the system considered in Fig. 2(b), but in the second frequency gap Eq. (24) is not satisfied and there is no resonance peak.

Next, we will examine the third condition (31). Here, we consider the case where the conditions (23) and (24) are satisfied. As a reference system, we examine the SL with the free surface again. In this system the phonon displacement of the surface-localized mode decays exponentially, as shown in Eq. (36). Conversely, the phonon amplitude increases exponentially away from the substrate-SL interface into SL

$$
\frac{\left|U_{0}\right|}{\left|U_{N}\right|}=(1+\varepsilon)^{-N} \cong e^{N|\varepsilon|},
$$

where $U_{0}$ and $U_{N}$ are the phonon displacement at the surface and the substrate-SL interface, respectively.

For the infinite SL (i.e., $N \rightarrow \infty$ ), this is an unphysical solution. However, this mode may exist in the finite-size SL. The transmission rate is defined as the ratio of energy flux, as shown in Eq. (3). In this definition, the acoustic impedance $Z_{S}$ and $Z_{D}$ for the substrate and detector materials are included. This means that the transmission coefficient may increase exponentially with $N$ if the acoustic impedance $Z_{D}$ is sufficiently smaller than $Z_{S}$. The maximum amplitude is restricted by the law of the conservation of energy,

$$
T=\frac{P_{D}}{P_{S}}=\frac{Z_{D}}{Z_{S}}|t(k)|^{2} \leqslant 1 .
$$

The condition, Eq. (30), is equivalent to the equation giving the upper limit of Eq. (38), because $|t|^{2}$ corresponds to $\left|U_{0} / U_{N}\right|^{2}$ in Eq. (37).

\section{CONCLUDING REMARKS}

In the present paper we studied the resonant transmission of phonons propagating through a SL-L interface. We calculated the phonon transmission rate and studied the peculiar resonance peak due to the vibrational mode localized near SL-L interface. The peak value depends on the number of periods $N$ of the superlattice, and this resonant feature disappears for large $N$ and also small $N$. Only for appropriate $N$ can we see the resonance. We derived the formula describing 
the condition for such a peak to be seen and discussed the physical meaning.

When the resonance of incident phonons with the localized modes occurs, the large temporal delay of phonon packets is predicted. ${ }^{14,18}$ To see the time delay expected in the SL-L system, the time development of the phonon packet propagating through the SL-L interface has been studied by numerical simulation. This result will be published elsewhere. $^{19}$

\section{ACKNOWLEDGMENTS}

The author acknowledges S. Tamura for reading the manuscript and giving valuable advice. A part of this work was done while the author was a visiting scientist at the Institute of Theoretical Physics, the Technical University of Dresden as Overseas Research Scholar of the Ministry of Education, Science, Sports and Culture of Japan. He acknowledges the hospitality of Dr. Dietmar Lehmann.
${ }^{1}$ For a compact review, see S. Tamura, Proceedings of the $3 r d$ International Conference on Phonon Physics and the 6th International Conference on Phonon Scattering in Condensed Matter, Heidelberg, 1989 (World Scientific, Singapore, 1990), p. 703.

${ }^{2}$ S. Tamura, D. C. Hurley, and J. P. Wolfe, Phys. Rev. B 38, 1427 (1988).

${ }^{3}$ V. Narayanamurti, H. L. Stormer, M. A. Chin, A. C. Gossard, and W. Wiegmann, Phys. Rev. Lett. 43, 2012 (1979).

${ }^{4}$ O. Koblinger, J. Mebert, E. Dittrich, S. Dottinger, W. Eisenmenger, P. V. Santos, and L. Ley, Phys. Rev. B 35, 9372 (1987).

${ }^{5}$ R. E. Camley, B. Djafari-Rouhani, L. Dobrzynski, and A. A. Maradudin, Phys. Rev. B 27, 7318 (1983).

${ }^{6}$ S. Tamura, Phys. Rev. B 39, 1261 (1989).

${ }^{7}$ H. T. Grahn, H. J. Maris, J. Tauc, and B. Abeles, Phys. Rev. B 38, 6066 (1988).

${ }^{8}$ H. J. Trodahl, P. V. Santos, G. V. M. Williams, and A. Bittar,
Phys. Rev. B 40, 8577 (1989).

${ }^{9}$ M. Giehler, T. Ruf, M. Cardona, and K. H. Ploog, Physica B 263-264, 489 (1999).

${ }^{10}$ M. Hammouchi, E. H. El Boudouti, A. Nougaoui, B. DjafariRouhani, M. L. H. Lahlaouti, A. Akjouj, and L. Dobrzynski, Phys. Rev. B 59, 1999 (1999).

${ }^{11}$ D. Bria, E. H. El Boudouti, and A. Nougaoui, B. Djafari-Rouhani, and V. R. Velasco, Phys. Rev. B 60, 2505 (1999).

${ }^{12}$ H. Kato, Phys. Rev. B 59, 11136 (1999).

${ }^{13}$ S. Mizuno and S. Tamura, Phys. Rev. B 45, 734 (1992).

${ }^{14}$ S. Mizuno and S. Tamura, Phys. Rev. B 53, 4549 (1996).

${ }^{15}$ F. Wallis, Phys. Rev. 105, 540 (1957).

${ }^{16}$ F. Wallis, Phys. Rev. 116, 302 (1959).

${ }^{17}$ J. Hori and T. Asahi, Prog. Theor. Phys. 31, 49 (1964).

${ }^{18}$ S. Mizuno and S. Tamura, Phys. Rev. B 50, 7708 (1994).

${ }^{19}$ K. Imamura, Y. Tanaka, S. Mizuno, and S. Tamura, J. Phys. Condens. Matter (to be published). 